\title{
Mandibular implant supported overdenture for an edentulous patient with less interridge distance using locator attachment- A case report
}

\author{
Sowmya Balakrishnan ${ }^{1, *}$, Rohit Raghavan ${ }^{2}$, Shajahan P.A ${ }^{3}$, Anil V Koruthu ${ }^{4}$, Mohammed Ramzi ${ }^{5}$ \\ 1,5 Assistant Professor, ${ }^{2}$ Professor and HOD, ${ }^{3}$ Professor, ${ }^{4}$ Associate Professor, Dept. of Prosthodontics, Royal Dental College, \\ Kerala, India
}

*Corresponding Author:

Email: sowmyabal@gmail.com

\begin{abstract}
Satisfying the edentulous patients with conventional complete denture has nowadays become a challenge to the prosthdontist as the patients have problems adapting to their denture especially in mandibular arch due to decreased retention, stability and difficulty in mastication. So a better treatment option for those patients is attachment retained implant supported overdenture. Overdenture helps in increasing the proprioception, retention, stability, comfort and confidence of the patient and decreasing the residual ridge resorption. Commonly used attachments used to retain implant supported overdenture are stud, bar, magnetic and telescopic attachment. This article presents a case report of implant supported overdenture for an edentulous patient with less interridge distance retained using locator attachment.
\end{abstract}

Keywords: Implant supported overdenture, Locator attachment, Less interridge distance.

\section{Introduction}

Prosthetic management of edentulous patients with conventional complete denture offer less retention, stability and comfort to the patient, particularly in mandibular arch. Conventional complete denture prosthesis needs frequent adjustments especially in mandibular arch because of greater bone resorption compared to maxilla. Overdenture is a complete or partial denture prosthesis which is supported partly by soft tissues and partly by retained natural teeth, roots or implants. Use of attachment retained implant supported overdenture increases the retention, stability, support, masticatory efficiency, proprioception and decreases the rate of ridge resorption which makes it an acceptable treatment option in mandibular arch. ${ }^{1}$

\section{Review of Literature of Various Attachments}

Attachments are mechanical devices for the fixation, retention and stabilization of the prosthesis. ${ }^{2}$ The selection of attachments for overdenture depends on various factors such as number of implants, interridge distance, type of prosthesis, amount of retention, patient expectation and cost factors. This case report describes the management of an edentulous patient with less interridge distance using mandibular implant supported overdenture with locator attachment. Various types of attachments used are stud attachment, locator attachment, bar attachment, magnetic attachment and telescopic attachment. ${ }^{3,4}$

\section{Stud Attachment}

Stud attachment consists of two parts. The stud (male component) which is attached to the fixture which comes as an implant abutment for overdenture prosthesis and the housing (female component) is fixed to the fitting surface of the denture. Stud attachments include O-ring attachment, Extraradicular attachment and ball attachment.

O-ring attachment consist of male component made of titanium and female component which is replacable metal ring. ERA attachment for implant supported overdenture is the ERA implant abutment for overdentures. These are available in two types first is the straight single piece abutment and second is the angulated abutment. In Ball attachment male portion is screwed to the implant and female part is attached to the fitting surface of the denture which offer different degrees of retention. ${ }^{5}$ Rodrique et al conducted a study of non axial forces on retention of o-ring attachment and found that it provide good retention when implants are parallel but retentive capacity and success rate is affected by implant angulations. ${ }^{6}$ Van kampen et al conducted a study on the retention of ball and socket, bar and clip and magnetic attachment and found that ball and socket provided the greatest retention followed by bar and clip and magnetic attachment. ${ }^{7}$

\section{Locator Attachment}

Locator attachment has got self aligning feature with dual retention (inner and outer).Reduced height of the attachment make it a choice in cases with less interidge distance. Locator female is attached to the fixture like abutment for overdenture and male part is attached to the fitting surface of the denture. Male portion consists of metal cap and the processing or retentive cap fitted inside the metal cap which offer different degrees of retention. ${ }^{8,9}$ Corado et al conducted a study on soft tissue interactions with different attachments and found that locator attachment has got better soft tissue interactions compared to bar attachments $^{10}$ 


\section{Magnetic Attachment}

Magnets are attached to the fitting surface of the denture and magnetic keeper is screwed to the implant. The retention of the magnetic attachment is less than ball attachment. ${ }^{11}$ Van Kampen conducted a study on retention of various attachments and found that retention is less for magnetic attachment than other attachments. ${ }^{7}$ Gillings et al in his article magnetic retention for complete and partial overdenture described advantages of magnetic attachments as it can be used in cases with less interridge distance, no $\mathrm{O}$ - rings to be replaced, lateral stress to implants are reduced than other attachments which increases the long term success of the prosthesis. ${ }^{12}$

\section{Bar Attachment}

Bar attachment is a metallic bar which splints two or more implants and a sleeve attached to the fitting surface of the denture which clips over the original bar. Bar can be prefabricated and custom made with single or multiple sleeves. The prefabricated bars are available in round, ovoid or rectangular cross section. ${ }^{13} \mathrm{Bar}$ provide some degree of rotation or resilient movement and spacers are provided to ensure a small gap between the sleeve and bar. Van Kampen et al in his study on retentive capacity of attachments described that bar and clip attachments provide better mechanical stability and more wear resistance compared to other attachments. ${ }^{7}$

\section{Telescopic Attachment}

Telescopic attachment which was commonly used with tooth supported overdenture has expanded its use to implant retained prosthesis. This is like double crown and sleeve coping. Retention is due to the frictional fit between crown and sleeve coping. ${ }^{14}$ Gotfredsen et al discussed the advantages of using these attachments as easy to maintain oral hygiene, provide good retention to prosthesis and disadvantages as technique sensitive, possibility of loss of retention due to mechanical wearing of copings. ${ }^{15}$

\section{Case Report}

A 58 year old male patient reported to the department of prosthodontics of Royal dental college with difficulty in chewing and poor facial appearance due to missing of all teeth in maxilla and mandible. Treatment planned was conventional complete denture in upper and lower arch. Patient was satisfied initially but later reported with decreased retention and stability of mandibular denture compared to maxilla during mastication. Considering the patient's expectation and less interridge distance better treatment option was attachment retained implant supported mandiblar overdenture using locator attachment.

\section{Locator Attachments}

Locator is a self aligning attachment for overdenture with inner and outer retention (dual). Locator attachment retentive caps come in different colours like black, clear, pink, green, orange, red which has got different degree of retention, reduced height of the attachment make it very useful for cases with limited interocclusal space and it can be attached to an existing old denture. ${ }^{8,9,16}$

\section{Salient Feature of locator attachments:}

1. Lowest Vertical Height: Height of the locator attachment is available with lowest vertical height compared to ball attachments so can be used in patients with less interridge distance.

2. Self-Locating Design: Even if the accurate alignment is not there the self aligining design allows the patient to easily seat their overdenture.

3. Dual Retention: unique dual retention provides greater retention surface area compared to other attachment

4. Rotation due to pivoting Action: The pivoting locator male has got a resilient connection for the prosthesis which helps without loss of retention ${ }^{17,18}$

\section{Retention and Angulation Versatility ${ }^{19}$}

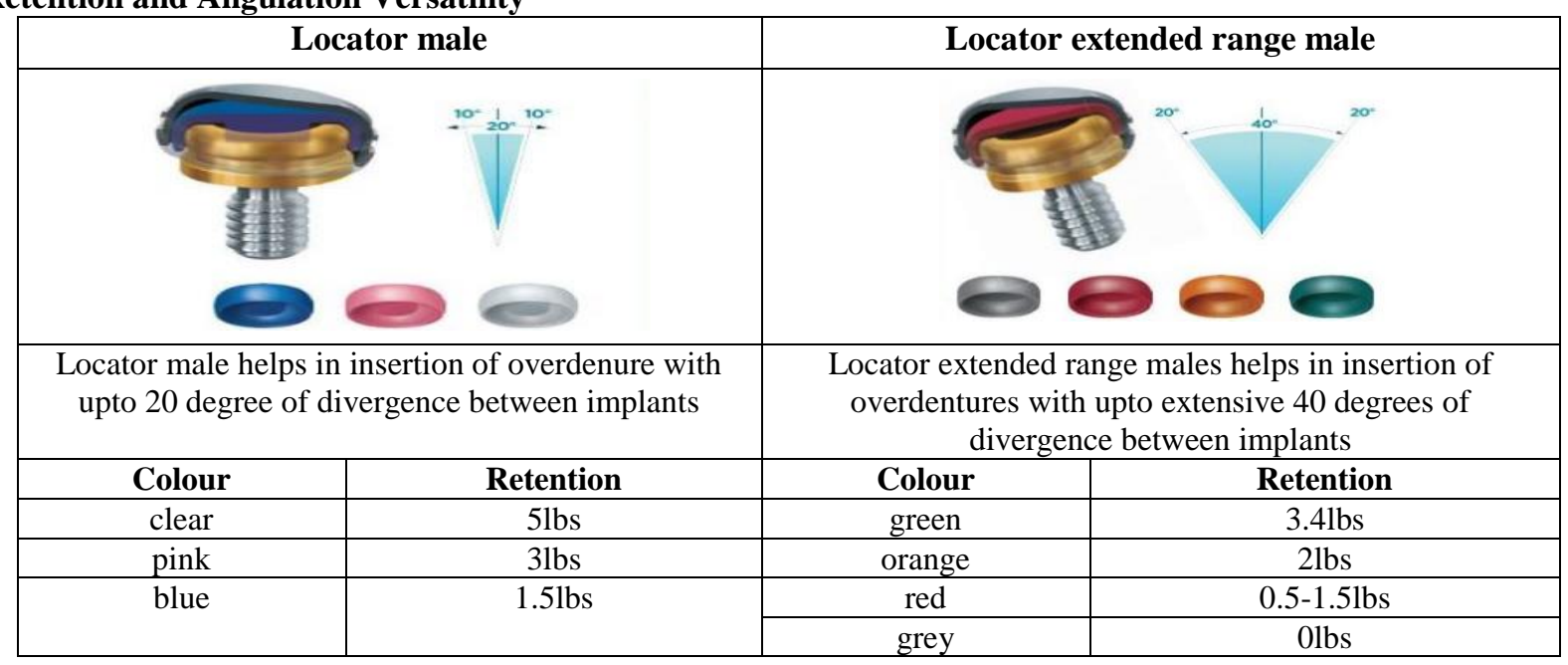


This case report is to be discussed in following three stages

1. Diagnosis and treatment planning

2. Surgical phase (Implant placement)

3. Prosthetic phase

\section{Diagnosis and Treatment Planning}

OPG and diagnostic impressions were made. Bone availability and quality of lower arch was evaluated by doing bone mapping intraorally and on the cast. (Fig. 1) Blood investigations were done to analyse the general health of the patient. Patient was informed about the possibility of an attachment retained implant supported overdenture as the best treatment option to solve his complaints. Two implants were planned in the canine region and locator attachment was used for retention of overdenture as the interidge distance was less.

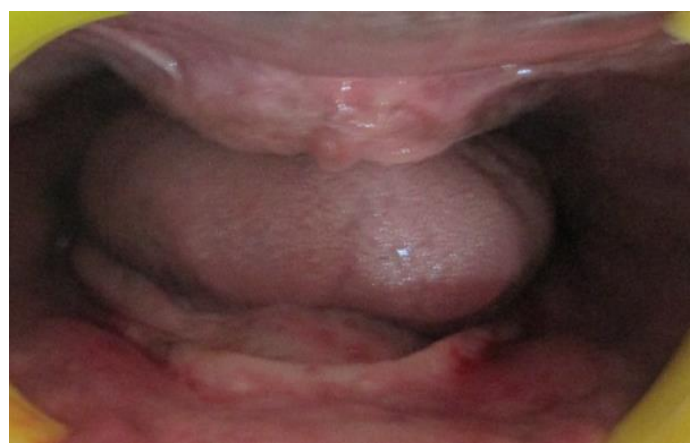

Fig. 1: Intra oral view of maxillary and mandibular arch

\section{Surgical Phase (Implant placement)}

Surgical stent was made using clear acrylic and two holes were made in the stent corresponding to the canine region. (Fig. 2) Two Nobel biocare replace select implants of size $4.3 \times 13 \mathrm{~mm}$ were placed in the canine region and cover screw was put and tightened. (Fig. 3). Patient was recalled after a week for suture removal and a review.

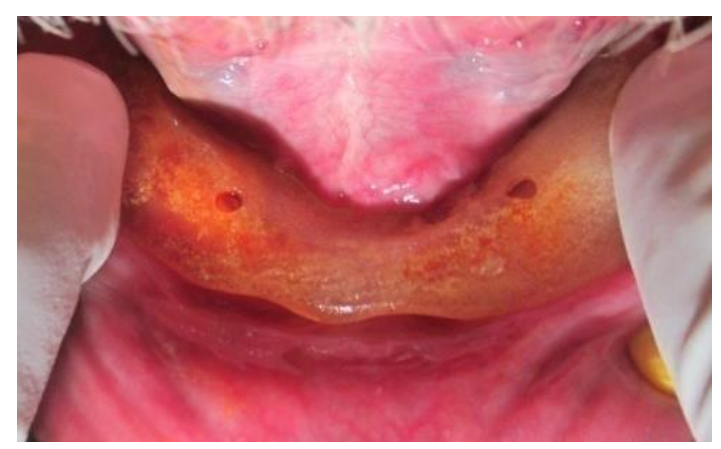

Fig. 2: Surgical stent made using clear acylic resin

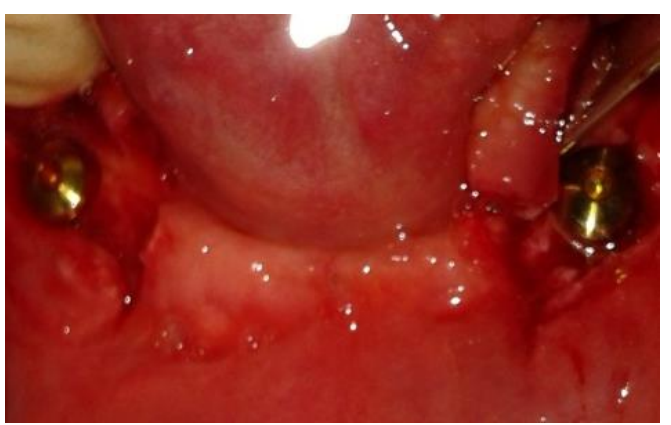

Fig. 3: Implant placement

\section{Prosthetic Phase}

Patient reported after 4 months of healing period for the prosththetic rehabilitation. A new conventional complete denture was fabricated in maxillary and mandibular arch. During jaw relation it was found that ball attachment cannot be used for retaining the overdenture because of less interridge distance, so locator attachment was selected for retention. Second stage surgery was performed and healing abutments were placed for getting proper gingival cuff. (Fig. 4)

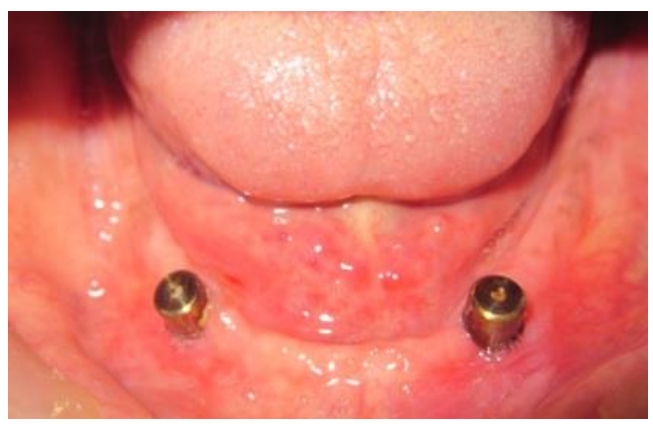

Fig. 4: Healing abutments were placed

Locator attachment has got dual retention, self aligning feature, various retentive attachment caps with different levels of retention. The pack consist of locator attachment, metal cap, white coloured block out spacer, black processing male cap and various coloured retentive attachment caps. After one week, healing abutments are then removed and locator attachment was placed.(Fig. 5) White coloured block out spacer was inserted above the locator attachment.. Then black processing cap was fitted into the metal cap and placed above the blockout spacer. (Fig. 6) Proper fitting of each component was assured. Then the implant positions were marked on the tissue surface of the denture and a small space for the self cure acrylic resin was trimmed and created. 


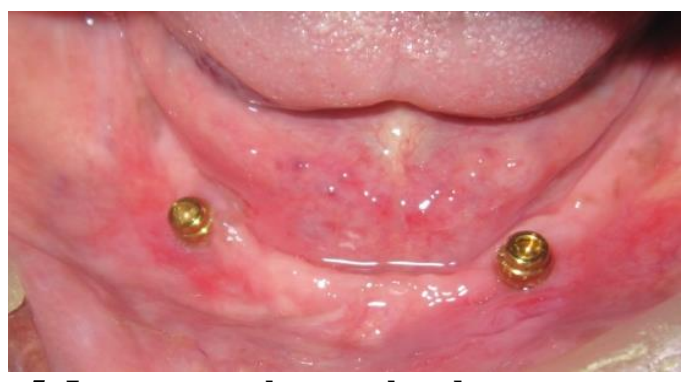

Fig. 5: Locator attachment placed

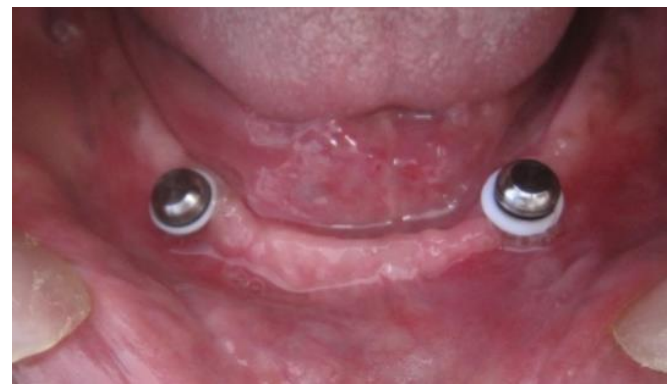

Fig. 6: Block out spacer and metal cap

Self cure acrylic was filled into that space in the denture and denture was placed on the mandibular arch, the metal cap and black processing cap was picked in the denture after the self cure sets. Black processing cap can then be removed and retentive attachment cap can be fitted and placed inside the metal cap based on the amount of retention required. (Fig $7 \& 8$ ) Finally the maxillary and mandibular denture is inserted inside the patients mouth and checked for occlusion and patient comfort. Patient was very satisfied with the denture because of increased retention, stability, masticatory efficiency and comfort. (Fig. 9)

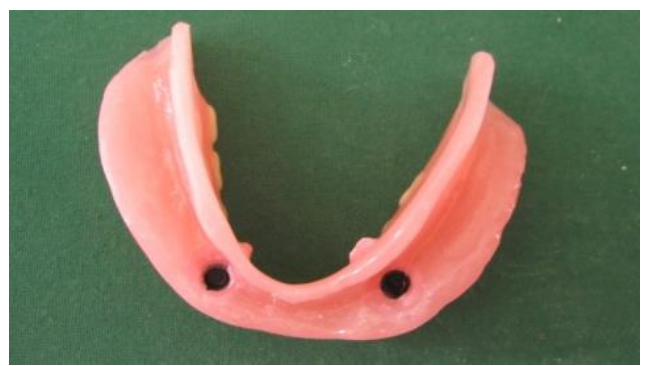

Fig. 7: Black processing cap picked in denture

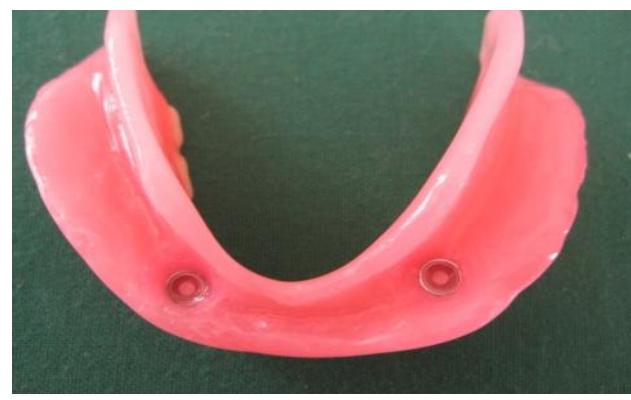

Fig. 8: Pink coloured retentive cap

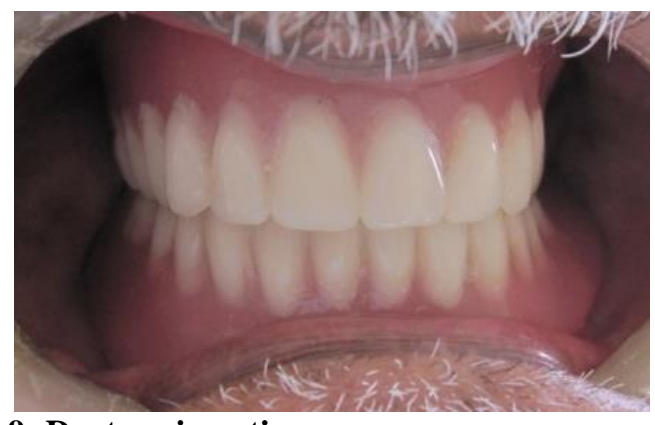

Fig. 9: Denture insertion

\section{Discussion}

Most of the edentulous patients have problem in adapting to conventional mandibular denture. Overdenture both tooth and implant supported proves to be a better treatment option because of proprioception, preservation of residual ridge, increased retention, stability and reversibility as it can be converted to conventional complete denture at any time. Implant supported attachment retained overdenture is the another best treatment option for those who are completely edentulous. Various types of attachments used are stud attachment, bar attachment, locator attachment, magnetic attachment and telescopic attachment. Attachments should be selected based on the number of implants, distance between ridges, type of prosthesis, degree of retention, patient expectation and cost factors. ${ }^{3,4}$ Hao-Sheng Chang conducted a $20 \mathrm{yr}$ retrospective study on long term survival rate of implant supported overdenture with different attachments and found that failure rate of implants with $\mathrm{O}$ ring attachment is less with locator attachment than ball and bar attachment. ${ }^{20}$ Evtimovska et al in his study proved that the locator can be used in cases with less interridge distance, it has got dual retention with various degree of retention caps, self aligning property and resilient connection with prosthesis. Locator attachment retentive caps come in various colours and has different retenive values. Extended range attachments are available and can be used to correct angulated implant upto $20^{\circ} .{ }^{21}$ Corado et al in his study compared two retention systems locator and bar and found same patient satisfaction with both and less of soft tissue interaction with locator attachment than bar attachment. ${ }^{10}$ Decreased vertical height makes it a good treatment option than ball attachment for cases with less inter ridge distance. ${ }^{22}$ Mohammed et al conducted a finite element study compared stress distribution of two attachment designs under implant supported overdenture found that the stress induced by locator and ball attachment on bone were found to be almost the same, but locator attachment was found to be superior to ball and socket attachment as the interval between maintenance sessions can be reduced with locator. ${ }^{23}$ Lyndon cooper et al discussed three case report of implant supported overdenture using locator attachment and found that edentulous patients were 
more satisfied with greater retention and stability, better esthetics and occlusion, improved maintenance and comfort and less soft tissue interaction. ${ }^{24}$

\section{Conclusion}

This case report described the management of an edentulous patient with less interridge distance with implant supported overdenture retained using locator attachment. This satisfied and increased the patient comfort and confidence in using the mandibular denture because of improved retention, stability, mastication and proprioception. Various factors like number of implants, interidge distance, type of prosthesis, amount of retention, patient expectation and cost factor is to be considered while selecting attachment for the success of the prosthesis.

\section{References}

1. Brewer AA, Morrow R M. overdentures $2^{\text {nd }}$ edition.CV Mosby St Louis 1980.

2. The Academy of Prosthodontics. The glossary of prosthodontic terms. J Prosthet Dent. 20015;94(1):10-92.

3. Alsiyabi AS, Felton DA, Cooper LF. The role of abutment attachment selection in resolving inadequate interarch distance: A clinical report. J Prosthodont. 2005;14:184-9.

4. Ahmed Yaseen Alqutaibi, Amal Fathy kaddah. Attachments used with implant supported overdenture. International Dental and Medical Journal of Advanced Research. 2016;2:1-5.

5. Winkler S, Piermatti J, Rothman A, Siamos G. An overview of the O-ring implant overdenture attachment: clinical reports. J Oral Implantol. 2002;28(2):82-86.

6. Rodrigues RC, Faria AC, Macedo AP, Sartori IA, de Mattos Mda G, Ribeiro RF. An in vitro study of non-axial forces upon the retention of an O-ring attachment. Clin Oral Implants Res. 2009;20:1314-9.

7. Van Kampen F, Cune M, van der Bilt A, Bosman F. Retention and postinsertion maintenance of bar-clip, ball and magnet attachments in mandibular implant overdenture treatment: An in vivo comparison aft er 3 months of function. Clin Oral Implants Res. 2003;14:720-6.

8. Evtimovska E, Masri R, Driscoll CF, Romberg E. The change in retentive values of locator attachments and hader clips over time. J Prosthodont. 2009:18(6):479483.

9. Pasciuta M, Grossmann Y, Finger IM A prosthetic solution to restoring the edentulous mandible with limited interarch space using an implant-tissue-supported overdenture: a clinical report. J Prosthet Dent. 2005;93(2):116-120.

10. Cordaro L, di Torresanto VM, Petricevic N, Jornet PR, Torsello F. Single unit attachments improve peri-implant soft tissue conditions in mandibular overdentures supported by four implants. Clin Oral Implants Res. 2013;24:536-42.

11. Preiskel HW. Overdentures made easy: a guide to implant and root supported prostheses. pp. 189-232

12. Gillings BRD. Magnetic retention for complete and partial overdentures. Part $1 \mathrm{~J}$ Prosthet Dent. 1981;45:484-491.

13. Wolfe R (1985) Symposium on semi precision attachments in removable partial dentures. Extracoronal attachments. Dent clin North Am. 29(1):185-198.

14. Langer Y, Langer A. Tooth-supported telescopic prostheses in compromised dentitions: a clinical report. $J$ Prosthet Dent. 2000;84(2):129-132.

15. Gotfredsen, K. and B. Holm. Implant-supported mandibular overdentures retained with ball or bar attachments: a randomized prospective 5-year study. Int $J$ Prosthodont. 2000;13:125-130.

16. Kleis WK, Kammerer PW, Hartmann S, Al-Nawas B, Wagner W. A comparison of three different attachment systems for mandibular two-implant overdentures: oneyear report. Clin Implant Dent Relat Res. 2010;12(3):209218.

17. Lyndon cooper, Arnold Rosen, Ibrahim Duqum. Implant supported overdenture options for the edentulous patient. International Dentistry SA. 13(1).

18. Prieskel HW.Precision Attachments in Prosthodontics. Overdenture and Telescopic prosthesis. Quintessence international 1985 vol 2.

19. Strumann. Locator male processing package (internet). Available from http://noticiasargentinas info/new/l/locator-male-processing-packagestraumann.awp

20. Hao Sheng Chang Yao Dung Hsieh Ming Lun Hsu. Long term survival of implant supported overdenture with various attachment sysyems. A 20 year Retrospective study. Journal of Dental Sciences. 2015;10(1):55-60.

21. Evtimovska E, Masri R, Driscoll CF, Romberg E. The change in retentive values of locator attachments and hader clips over time. J Prosthodont. 2009;18:479-83.

22. Kleis WK, Kämmerer PW, Hartmann S, Al-Nawas B, Wagner W.A comparison of three different attachment systems for mandibular two-implant overdentures: one year report. Clin Implant Dent Relat Res. 2010;12:20918.

23. Mohammed I El Anwar. Comparison between two low profile attachment for implant mandibular overdenture. Journal of genetic engineering and biotechnology. 2014;12(1);45-51.

24. Lyndon Cooper, Arnold Rosen, Ibrahim Duqum. Implant supported overdenture options for the edentulous patients. International Dentistry SA. V13;(1):12-16. 